\title{
A Structural Approach to Enhancing WordNet with Conceptual Frame Semantics
}

\author{
Ivelina Stoyanova \\ Institute for Bulgarian Language \\ Bulgarian Academy of Sciences \\ iva@dcl.bas.bg
}

\author{
Svetlozara Leseva \\ Institute for Bulgarian Language \\ Bulgarian Academy of Sciences \\ zarka@dcl.bas.bg
}

\begin{abstract}
This paper outlines procedures for enhancing WordNet with conceptual information from FrameNet. The mapping of the two resources is non-trivial. We define a number of techniques for the validation of the consistency of the mapping and the extension of its coverage which make use of the structure of both resources and the systematic relations between synsets in WordNet and between frames in FrameNet, as well as between synsets and frames).

We present a case study on causativity, a relation which provides enhancement complementary to the one using hierarchical relations, by means of linking in a systematic way large parts of the lexicon. We show how consistency checks and denser relations may be implemented on the basis of this relation.

We, then, propose new frames based on causative-inchoative correspondences and in conclusion touch on the possibilities for defining new frames based on the types of specialisation that takes place from parent to child synset.
\end{abstract}

\section{Introduction}

The research presented in this paper aims at enhancing WordNet with information about the conceptual structure of verbs based on the mappings between WordNet (WN) and FrameNet (FN). This information includes description of the conceptual elements that receive expression as verbs' arguments and adjuncts as well as the selectional restrictions imposed on these elements.

Our approach relies on the structural features of each of the resources as well as on various kinds of linguistic analysis. The proposed enhancement is directed to: (a) improving the quality of existing mappings; (b) expanding the mappings' coverage; (c) enhancing the description of frames with additional information obtained from WN; (d) proposing structural improvements on the resources based on systemic features (e.g., causativity), including the definition of new conceptual frames; and (e) suggesting further procedures for verification and improvements of precision.

The described methodology is semi-automatic (automatic assignment complemented by manual verification at several stages), but its contribution consists in the devising of a set of procedures to a structured and consistent enrichment of the two resources, which presents an important step towards its automatisation. The enhanced resources will be made available to the research community. As the description of verbs' conceptual structure is largely language independent, the enriched description is applicable cross-linguistically.

\section{Prerequisites and Motivation}

\subsection{Linguistic Resources}

WN (Miller, 1995; Fellbaum, 1998) is a large lexical database that represents comprehensively the conceptual and lexical knowledge in the form of a network whose nodes denote cognitive synonyms (synsets) interconnected through a number of conceptual-semantic and lexical relations. The main relation that determines WN's structure (as reflected in the hierarchical treelike organisation of nouns and verbs) is a relation of inheritance of conceptual and lexical features between synsets. The respective pairs of synsets are linked through the relation of hypernymy/hyponymy.

FN (Baker et al., 1998; Baker, 2008) represents lexical and conceptual knowledge couched in the apparatus of frame semantics. Frames are concep- 
tual structures describing particular types of objects, situations, or events along with their components, called frame elements, or FEs (Baker et al., 1998; Baker and Ruppenhofer, 2002; Ruppenhofer et al., 2016). Depending on their status, FEs may be core, peripheral or extra-thematic, cf. Ruppenhofer et al. (2016). For our purposes, we deal particularly with core FEs, which instantiate conceptually necessary components of a frame, and which in their particular configuration make a frame unique and different from other frames. To a lesser degree we touch upon peripheral FEs, which mark notions such as Time, Place, Manner, Means, among others and may be instantiated in any semantically appropriate frame. A Lexical Unit (LU) in FN is a pairing of a word with a meaning; each LU is associated with a frame describing its conceptual semantics.

\subsection{Structural Properties of WN and FN}

FN frames are related into a netlike structure through a number of frame-to-frame relations part of which also provide a hierarchical organisation. These relations are presented in detail in Leseva and Stoyanova (2019); below, we just sum up those used in the procedures we propose.

Inheritance (Is Inherited by $\leftrightarrow$ Inherits from) is the strongest and most prominent relation in FN, which posits a relationship between a more general (parent) frame and a more specific (child) frame so that the child frame elaborates on the parent frame in such a way that each semantic fact about the parent must correspond to an equally or more specific fact about the child (Ruppenhofer et al., 2016, p. 81-82). By definition, Inheritance corresponds to the relation of hypernymy/hyponymy in WordNet. In an ideal setting, the hyponyms should be instantiations of the hypernym's frame or of a more specific frame that inherits from the hypernym's frame. For instance, the frame Execution Inherits from Killing and is assigned to the WN synset \{execute: 1 , put to death: 1$\}$ - a hyponym of $\{$ kill: 1$\}$, which is assigned the frame Killing.

As the two resources have been developed independently, their relational structure is distinct, one of the major differences being that there are other frame-to-frame relations that to various degrees embody the notion and features of inheritance; we use (some of) them in the definition of the procedures proposed in Section 4.1.
The first one is Using (Is Used by $\leftrightarrow$ Uses), a frame-to-frame relation defined as a relationship between two frames where the first one makes reference in a very general kind of way to the structure of a more abstract, schematic frame (Ruppenhofer et al., 2016, p. 83); it may be viewed as a kind of weak inheritance (Petruck, 2015) where only some of the FEs in the parent frame have a corresponding entity in the child frame, and if such exist, they are more specific (Petruck and de Melo, 2012). Thus, the frame Arranging Uses the frame Placing and the two frames share the FEs Agent and Theme, while the more specific FE Configuration in the frame Arranging corresponds to the more general FE Goal in the frame Placing; the first frame is exemplified by the synset $\{$ arrange: 1 , set up:5\} which is a hyponym of \{put:1, set:1, place:1, pose:5\} whose assigned frame is the more general frame Placing.

We consider two more relations although they align with the notion of inheritance only marginally. Perspective (Is Perspectivized in $\leftrightarrow$ Perspective on) is defined as a relation which indicates that a given situation viewed as neutral may be further specified by means of perspectivised frames representing different possible points-ofview on this neutral situation (Ruppenhofer et al., 2016, p. 82). Subframe (Has Subframe(s) $\leftrightarrow$ Subframe of) is a relation between a complex frame referring to sequences of states and transitions (each of which can itself be separately described as a frame) and the frames denoting these states or transitions (Ruppenhofer et al., 2016, p. 83-84).

These hierarchical relations are the basis for part of the procedures outlined in Section 4.

\subsection{Causativity in WN and FN}

Causativity (Is Caused by $\leftrightarrow$ Causative of) is a systematic non-inheritance relation where one of the frames represents the causative counterpart of the other, stative or inchoative, frame (Ruppenhofer et al., 2016, p. 85). Causativity corresponds straightforwardly to the WN relation causes, although this correspondence is exhibited in only a small number of cases (30 pairs) - the relation has not been implemented consistently neither in FN, nor in WN even in clear-cut parts of the lexicon such as those described by the hypernym trees with the roots $\{$ change: 1, alter:1, modify: 3$\}$ ('cause to change; make different; cause a transformation') and \{change:2\} ('undergo a change; 
become different in essence; losing one's or its original nature').

\section{Existing Mappings of WN and FN}

Previous efforts at linking WN and FN include Shi and Mihalcea (2005), Baker and Fellbaum (2009), WordFrameNet ${ }^{1}$ (Laparra and Rigau, 2009, 2010), MapNet $^{2}$ (Tonelli and Pighin, 2009), and more enhanced proposals, such as the system Semlink ${ }^{3}$ (Palmer, 2009) which brings together WN, FN and VerbNet with PropBank, and its follow-up Semlink+ that brings in mapping to Ontonotes (Palmer et al., 2014). Some procedures for automatically extending the mapping, are presented by Leseva et al. (2018) and a more thorough overview may be found in Leseva and Stoyanova (2019).

Whereas these efforts have resulted in the creation of databases of integrated semantic knowledge, most of them deal with mapping of the units of the original resources to each other - FN LUs and WN synset members (literals), LU definitions and synset glosses, etc. Such a methodology is able to perform mapping in those cases where there is a correspondence between LUs and literals with equivalent or close meaning, but would fail where such correspondence is missing. With 155,287 synonyms in 117,659 synsets and more than 246,577 relations, of which 91,631 are instances of the hypernymy relation ${ }^{4}$ as compared with 13,640 LUs and 1,875 frame-to-frame relations ${ }^{5}$ in FN, the discrepancy in the size of the data is reflected in the limited coverage of the mappings between synsets and frames. To the best of our knowledge, no further checks and verification have been performed on the mappings, as well.

The approach that we propose in addition to the lexical mapping of units deals with exploring and taking into account the relational structure of the resources (especially the structure of $\mathrm{WN}$ ), particularly the relation of inheritance which ensures the propagation of conceptual and linguistic features down the trees. We employ features of the relational structure in the definition of procedures for the augmentation of the mapping cov-

\footnotetext{
${ }^{1}$ http: //adimen.si.ehu.es/web/ WordFrameNet

${ }^{2}$ https://hlt-nlp.fbk.eu/technologies/ mapnet

${ }^{3}$ https: / / verbs. colorado.edu/semlink/

${ }^{4}$ https: //wordnet.princeton.edu/ documentation/wnstats $7 w n$

${ }^{5}$ https://framenet.icsi.berkeley.edu/ fndrupal/current_status
}

erage which are aimed at: (i) discovering existing but unmapped relations between synset members and FN frames; and (ii) transferring frames between synsets through relations of inheritance derived from WN and FN.

\section{Enhancing WN Mappings to FN}

As noted above, the proposed approach combines the features used in the direct mapping with the structural properties of $\mathrm{WN}$ and $\mathrm{FN}$ - particularly, the inheritance relations existing between hypernyms and hyponyms in WN and the inheritance (and other similar relations) that determine the hierarchical structure of FN. As shown in Leseva and Stoyanova (2019), although the relations in the two resources have different number and scope, part of them are grounded in similar universal assumptions which leads to partial overlap, depending on their definition and the specificities of the information in the resources.

Apart from the correspondences between FN's Inheritance and other relations and the $\mathrm{WN}$ hypernymy relation, there are other systematic structural relations which can be applied for the purpose of enhancing the resources. Notable examples are the Causativity relation between frames in $\mathrm{FN}$ and the causes relation defined between causative and stative or inchoative verbs in WN (cf. Section 5).

\subsection{Expanding Mappings based on Hierarchical Relations in WordNet}

Our work relies on the assumption that in a taxonomic structure such as WN subordinate nodes inherit the properties of their superordinates, i.e. a hyponym elaborates on the meaning of its hypernym and shares its conceptual and linguistic properties. We propose that if a WN synset instantiates a particular FN frame, its hyponyms should (ideally) instantiate the same or a more specific frame which may or may not hold a(n) (inheritance) relation with the more general frame.

This assumption allows us to suggest that in the cases where we are not able to assign a FN frame due to the fact that the coverage of the two resources is non-overlapping and/or other mapping procedures fail, we may resort to assigning the frame of a hypernym to its hyponyms; at worst, the semantic representation will be too general.

There are 14,103 verb synsets in WN, which, unlike nouns that all have a common root, are organised in 566 separate trees. Initially, FN frames 
have been assigned to a total of 4,306 synsets out of which 264 are root synsets. In order to improve the quality of the existing mappings and to expand the coverage we performed the following steps: (i) manual verification of the frames assigned to root synsets (resulting in 75 corrected mappings); (ii) semi-automatic assignment of valid frames to selected root synsets with a large number of hyponyms (27 roots); (iii) assignment of a hypernym's frame to its hyponyms in the cases where a hyponym is not directly mapped to FN frames, thus obtaining an extended coverage of 13,226 verb synsets with an assigned FN frame; (iv) definition of further procedures to the end of improving the quality of this assignment (section 4.2).

\subsection{Selection of Frames based on the $\mathrm{FN}$ and the WN Structure}

We devised two types of procedures aimed at obtaining a more specific mapping: (i) procedures that make use of the conceptual and lexical information and the relational structure in FN; (ii) procedures employing the conceptual and lexical information and the relational structure in $\mathrm{WN}$.

As noted above, the first step of assigning a FN frame to a WN synset is transferring the frame assigned to the synset's direct or inherited hypernym. The frame so assigned may either appropriately describe the conceptual structure of the literals in the synset, or it may provide a more general description than an optimally informative one. We therefore view this as a default assignment on the basis of which we try to elaborate to the end of discovering a more suitable or specific frame to which to map the synset. When such a frame is found, we validate it manually and assign it to the hyponyms of the synset under discussion, overriding the more general frame as in Example 1.

Example 1. Synset: eng-30-00047945-v \{dress:6; clothe:1; enclothe:1; garment: 1$\}$ 'provide with clothes or put clothes on'

Assigned FR from hypernym: Undergo_change Suggested FR: Dressing (transferred automatically to 13 out of 15 hyponyms such as $\{$ corset: 1$\}$ 'dress with a corset', $\{$ vest: 1$\}$ 'dress with a vest', \{overdress:2\} 'dress too warmly')

Below, we describe the procedures proposed and how they make use of the relational structure of FN and WN and the following components of the description in the two resources, in particular: (i) WN literals (and synsets) and synset-to-synset relations - especially the hypernymy relation, as well as the relations between synsets with a common hypernym (i.e., sister synsets); and (ii) LUs from a particular FN frame (the verbs listed as instantiations of a given frame), the hierarchical frame-to-frame relations: Inheritance, Uses, Subframe, and Perspective, as well as the relation between two frames inheriting from the same frame (i.e., sister frames).

For a synset assigned a frame inherited from its hypernym, we apply the following procedures:

(1) Literal-LU correspondence using FN relations: We check whether any of the synset literals appears as a LU in: (a) the assigned frame (to confirm its validity); (b) more specific frames the frame under discussion is linked to by means of any of the considered frame-to-frame relations (to make it more precise); (c) the sister frames of the assigned frame.

Example 2. Synset: eng-30-00540946-v \{extend:8; expand:4\} 'expand the influence of' Assigned FR from hypernym: Cause_change Suggested FR from (1b): Change_event_duration (LU: extend)

Suggested FR from (1c): Cause_expansion (LU: expand)

(2) Literal-LU correspondence using $\mathbf{W N}$ relations: We check whether any of the synset literals appears as a LU in: (a) any of the frames assigned to its hyponyms; (b) any of the frames related to the frames in (a) through frame-to-frame relations; and (c) any of the frames assigned to its sister synsets.

Example 3. Synset: eng-30-00223374-v \{bolster: 1; bolster up:1\} 'support and strengthen' Assigned FR from hypernym: Cause_change Suggested FR from (2c): Supporting (LU: bolster)

(3) General literal-LU correspondence: We check whether any of the synset literals appears as a LU in any other frame in FN.

Example 4. Synset: eng-30-00544936-v $\{$ exalt: 1$\}$ 'raise in rank, character, or status' Assigned FR from hypernym: Cause_change Suggested FR from (3): Judgment (LU: exalt)

(4) Keywords: We use keywords (words contained in the FN frame name, plus their derivatives collected from WN through the eng_derivative relation), to identify synset literals and/or definitions containing these keywords as candidates to be as- 
signed the frame in question.

Example 5. Synset: eng-30-00448864-v \{clean out:1; clear out:1\} 'empty completely' Assigned FR from hypernym: Cause_change Suggested FR from (4): Emptying (keyword empty found in gloss)

(5) Direct similarity: We check the similarity between the gloss of a verb synset and FN LU definitions (even when there is no correspondence between literals and LUs) to identify candidate frames for a given verb synset. We separate: (i) suggested frames related to the one assigned from the hypernym, which are given higher priority; (ii) unrelated suggestions.

Example 6. Synset: eng-30-02514187-v \{gloss over:1; skate over:1; smooth over:1; slur over:1; skimp over:1\} 'treat hurriedly or avoid dealing with properly'

Assigned FR from hypernym: Intentionally_act Suggested FR from (5): Avoiding (which Inherits from Intentionally_act); similarity with the definition of LU skirt.v 'avoid dealing with'

(6) Indirect similarity: We check the similarity between the glosses of synsets derivationally related to the verb under discussion (as well as the glosses of their hypernyms, which are considered their closest semantic generalisation) and FN LU definitions to identify candidate frames for the verb synset. We separate: (i) suggested frames related to the one assigned from the hypernym, which are given higher priority; (ii) unrelated suggestions.

Example 7. Synset: eng-30-00831651-v $\{$ warn: 1$\}$ 'notify of danger, potential harm, risk' Assigned frame from hypernym: Telling Derivationally related synset: eng-3007224151-n \{warning: 1\} 'a message informing of danger'

Suggested FR from (6): Warning (Inherits from Telling); similarity with the gloss of LU alert.n 'a message to inform someone of danger; a warning'

Similarity in procedures (5) and (6) is calculated as a cumulative measure based on coinciding terms in the two definitions. Scores of similarity between two words are highest for full match and lower when stemming is applied. Short words (up to length 3) are disregarded and longer words are given more weight. The final score is normalised by the length (in words) of the definitions.

Through these steps 9,341 new suggestions of

\begin{tabular}{|l|l|l|l|}
\hline Procedure & $\begin{array}{l}\text { \# 1-step } \\
\text { transfers }\end{array}$ & $\begin{array}{l}\text { \# 2-step } \\
\text { transfers }\end{array}$ & $\begin{array}{l}\text { \# 3+ step } \\
\text { transfers }\end{array}$ \\
\hline$(1)$ & 516 & 231 & 121 \\
\hline$(2)$ & 460 & 41 & 17 \\
\hline$(3)$ & 1,701 & 859 & 145 \\
\hline$(4)$ & 1,088 & 612 & 27 \\
\hline$(5)$ & 1,175 & 526 & 202 \\
\hline$(6)$ & 1,009 & 417 & 194 \\
\hline \hline $\begin{array}{l}\text { Unique } \\
\text { synsets }\end{array}$ & 3,957 & 1,388 & 316 \\
\hline
\end{tabular}

Table 1: Distribution of frames suggested for synsets with automatic frame assignments from the hypernym (rows (1)-(6) include multiple suggestions for the same synset)

more specific or other possible frames have been made for 5,661 synsets with automatically transferred hypernym frames - Table 1 shows the distribution of the new suggestions in terms of the types of procedures that have been applied and the distance of the synset from the hypernym whose frame has been inherited.

\subsection{Discussion on Evaluation}

These suggestions need to be manually verified as so far no reliable fully automated verification procedure has been established. Since the main objective is to discover, or suggest, a more precise frame than the one assigned from the hypernym, which is not necessarily wrong but rather may be too general, such evaluation needs to measure the degree of relevance as opposed to precision. Furthermore, it will be highly dependent on the granularity of the frames and their hierarchical organisation. Designing such a measure and its automatisation, if at all achievable, is beyond the scope of this work.

Suggestions, although non-definitive, provide useful pointers to candidate frames and thus are valuable in assisting the manual selection of frames. Only in 203 cases are there multiple suggestions as a result of the procedures, out of which in 177 cases 5 or more different frames are suggested. There are 1,056 synsets for which a suggestion is confirmed at least 2 times from the repeated application of the same or different procedures, out of which 265 cases are confirmed 5 or more times.

Given the task, human judgment is indispensable, especially for frames assigned to synsets 
higher in the tree as errors propagate down and may result in multiple wrong assignments.

\section{Causativity and Inchoativity as a Systemic Structural Feature}

Another direction of expanding the mappings and verifying the information in both $\mathrm{FN}$ and $\mathrm{WN}$ is by employing systematic semantic relations such as causativity. It is a non-hierarchical relation that links stative (e.g. $\{l i e: 2\}$ 'be lying, be prostrate; be in a horizontal position') or inchoative (\{lie down:1, lie: 7$\}$ 'assume a reclining position') verbs with their causative (\{lay:2, put down:2, repose:5\} 'put in a horizontal position') counterparts. The relation provides enhancement complementary to the one using hierarchical relations described above and links in a systematic way large parts of the lexicon.

A considerable part of causative and noncausative pairs are formed with the same root and are thus morphologically similar or identical, e.g. EN change - change; RO schimba - schimba; BG promenyam - promenyam se, which makes them easier to identify. Nevertheless, as noted above, causativity is not consistently encoded in $\mathrm{WN}$, and neither is it fully implemented in FN where we have spotted a number of instances of inchoative/stative or causative frames lacking a counterpart in the opposite domain. This means that the verbs instantiating them cannot be appropriately described in FN. Respectively, the mapping of literals instantiating non-defined frames will result in failure of assignment or wrong assignment.

Causativity also has an important application in $\mathrm{WN}$ and FN data validation and expansion: exploring the assignment of frames from $\mathrm{FN}$ to synsets enables us to check the consistency of assignments, by adopting the following logic: (i) in a tree whose root is a causative synset, all the descendants must be assigned a causative frame; (ii) in a tree with an inchoative/stative root all the descendants must be inchoative/stative; (iii) the pairs of causative-non-causative synsets from corresponding trees should be connected to each other through the WN causes relation in a consistent way; (iv) the respective pair of causative-noncausative frames assigned to such a pair of synsets should also be related via the Is Causative of relation in FN. The opposite signals either wrong assignment of a frame or inconsistency either in the WN data, that is - the encoding of a stative or in-

\begin{tabular}{|l|l|l|}
\hline & $\begin{array}{l}\text { Causative } \\
\text { change:1; }\end{array}$ & $\begin{array}{l}\text { Non-causative } \\
\text { change:2; }\end{array}$ \\
\hline From FN & 241 & 251 \\
\hline Direct hypernym & 910 & 624 \\
\hline Indirect hypernym & 577 & 469 \\
\hline Total & 1,728 & 1,344 \\
\hline \hline General frame* & 719 & 561 \\
\hline in \% & $41.6 \%$ & $41.7 \%$ \\
\hline
\end{tabular}

Table 2: Analysed data with respect to causativity (* assignments of the most general frame Cause _change for the causative and Undergo change for the non-causative)

choative verb in a causative tree or vice versa, or in the FN data - missing or wrong relation between frames, undefined frames, etc.

Sections 5.1 and 5.2 describe the procedures for exploring pairs of causative-non-causative trees and extracting information enabling the validation of assigned frames, as well as the increase of the density of causativity relations within FN and WN. Section 5.3 deals with the formulation of new causative or stative and/or inchoative frames.

\subsection{Analysis and Consistency Checks}

We have extracted two separate $\mathrm{WN}$ trees from two root synsets connected by the causes relation (see Table 2): (1) eng-30-00126264$\mathrm{v}$ \{change: 1 ; alter: $1 ;$ modify: 3$\}$, assigned the frame Cause_change; and its corresponding non-causative counterpart (2) eng-30-00109660-v \{change:2\}, assigned the frame Undergo_change.

The checks for consistency with regards to (i)(iv) above, included the following procedures:

(1) Identifying non-causative synsets in the causative tree and causative synsets in the noncausative tree. These mismatches are identified by pattern matching of the gloss or by analysis aimed at establishing whether the manually assigned frame contradicts the position in the tree. 9 such cases have been found in the causative tree (e.g., eng-30-00416880-v \{even:6; even out:2\} 'become even or more even'). Pattern-matching in the non-causative tree proved to be unreliable. It identified 120 cases of 'make' or 'cause' in the gloss, but only a small number of them were causative synsets (e.g., eng-30-00330565-v \{break up:3; disperse: 1 ; scatter: 1$\}$ 'cause to separate'). We propose moving each wrongly placed synset (and the subtree rooting from it) to the rel- 
evant tree and attaching it to its real hypernym.

Furthermore, there are synsets which combine the causative and the non-causative meaning and thus, create inconsistency in the WN structure. We identify such synsets by pattern matching of the gloss since they usually have glosses such as 'make or become', 'cause or become', 'cause or undergo'. There are 7 cases in the causative (e.g., eng-30-01253468-v \{coarsen: 2$\}$ 'make or become coarse or coarser') and 5 in the non-causative tree (e.g., eng-30-00280532$\mathrm{v}\{$ blacken:1; melanize:1\} 'make or become black'). We propose that such synsets are split into two and placed at the respective positions in the relevant trees. This is an optimal solution as these concepts are not necessarily expressed by the same lexeme cross-linguistically, and such a split improves the consistency of WN.

(2) Identifying non-causative frames assigned to synsets in the causative tree and causative frames assigned to synsets in the non-causative tree. A causative frame is identified based on: keywords such as 'cause' or 'make' in its name or its definition; Agent or Cause/Causer FEs in its conceptual structure; its position as the first member in a Is Causative of relation, etc. A noncausative frame is identified based on: keywords such as 'become' or 'undergo'; lack of Agent or Cause/Causer FEs in its structure; its position as the second member of an Is Causative of relation.

We found 7 non-causative frames in the causative tree (e.g., eng-30-02190188-v \{quieten:3; hush:5; quiet:9; quiesce:1; quiet down:1; pipe down:1\} 'become quiet or quieter', Frame: Becoming_silent) and 61 causative frames in the non-causative tree (e.g., eng-30-00339085$\mathrm{v}\{$ crush: 1$\}$ 'break into small pieces', Frame: Cause_to_fragment). These are clearly either errors in the frame assignment or wrongly encoded synsets as discussed in (1).

(3) Identifying synset pairs connected by the causes relation in WN where the causative synset is assigned a non-causative frame or vice versa. Section 5.2 deals with the enrichment of the two resources with instances of the causative relation.

\subsection{Densifying Causative Relations in WN and FN}

The causative tree stemming from $\{$ change: 1$\}$ and the non-causative one stemming from $\{$ change: 2$\}$ were aligned using the WN causes relation, result- ing in 47 pairs of corresponding synsets - one in each tree. A set of consistency checks showed that there are no crossing relations (i.e., no instances where for a causative hypernym $C_{1}$ and its hyponym $C_{2}$, and a non-causative hypernym $N_{1}$ and its hyponym $N_{2}, C_{1}$ causes $N_{2}$ and $C_{2}$ causes $N_{1}$ ).

Further procedures were proposed to discover pairs of corresponding causative and noncausative synsets unrelated through the causative relation. These are based on pattern matching of the definition and/or on measuring similarity, as well as on an analysis of the synsets position in the WN tree structure, the causative relations in which their sisters, hypernym and hyponyms enter, and the frames assigned to them. On the basis of these linguistic features we have identified 673 possible causative relations between pairs of synsets in the two corresponding trees. After manual validation they may be used to create a more dense structure of causative relations in $\mathrm{WN}$, as well as to extend them to frame-to-frame relations in $\mathrm{FN}$.

\subsection{Suggesting New Frames}

New frames are suggested where a suitable causative or non-causative frame is not defined in FN to match its existing counterpart. The missing one is defined using the conceptual description of the available frame. Consider the synset $\{$ age:3\} 'make older': we assign it the frame Cause_change and then try to acquire additional classificatory information and, possibly, to find a more specific frame by applying the remaining procedures. We confirm that the synset's meaning is causative through the keyword procedure (cf. Section 4.2). Another mapping procedure suggests Aging as the corresponding frame. Aging is a non-causative frame denoting the meaning of an entity undergoing a particular kind of change (see Example 8). Since Aging does not have a causative counterpart in $\mathrm{FN}$, we posit such a frame, Cause_to_age. The conceptual structure of stative/inchoative and causative counterparts is distinguished by the presence of a causative subevent in the latter (Van Valin Jr. and LaPolla, 1997 , p. 109) which is associated with a causative (Agent or an Agent-like) participant (FE). Thus, in the discussed example Cause_to_age is derived from Aging by enriching the set of Aging's FEs with the frame elements Cause and Agent. In addition, we posit a Causative_of relation between Cause_to_age and Aging. In general, causative 
frames inherit from the abstract frame Transitive_action so we define an Inheritance relation between Transitive_action and Cause_to_age. In such a way the newly-defined relation is integrated into the FN relational structure.

Example 8. Frame: Cause_to_age Core frame elements: Agent/Cause; Entity FN definition: An Agent or Cause causes an Entity to undergo a change in age typically associated with some deterioration or change in state.

Example synsets: $\{$ age: 3$\}$ 'make older'

FN relation: Inherits_from

Frame: Transitive_action

Core frame elements: Agent/Cause; Patient

Frame definition: An Agent or Cause affects a Patient.

FN relation: Is_Causative_of

Frame: Aging

Core frame elements: Entity

Frame definition: An Entity is undergoing a change in age typically associated with some deterioration or change in state.

Example synset: \{senesce:1, age:2, get on:7, mature:5, maturate: 2$\}$ 'grow old or older'

The domain of causativity provides an approach at symmetricising large parts of the lexicon both at a horizontal level (same level lexemes in a taxonomical hierarchy) and in depth as the improvements in the higher levels of the lexicon influence the deeper levels as reflected in the procedure of assigning relations by inheritance described in 4.1.

\section{Frame Specialisation and Relations}

The observations on hierarchical relations, especially on the more populated ones, such as Inheritance, Using and See_also, shed light on the specialisation that takes place from parent to child in the taxonomic (inheritance) hierarchy. The changes in the causativity domain deal with including/excluding FEs that correspond to causative subevents in the event structure. The modifications that occur in the conceptual and semantic structure include, but are not limited to the following:

- Reducing the number of core frame elements by incorporating one of them in the verb's meaning, e.g. $\{$ whip: 4$\}$ incorporates the peripheral FE Instrument ('whip') of $\{$ strike: 1$\}$ in the frame Cause harm assigned to both;

- Reducing the scope of the frame through imposing more strict selectional restrictions on the
FEs, e.g. $\{$ drive: 1$\}$ (Operate_vehicle) as a hyponym of \{operate:3\} (Operating_a_system) applies only to land vehicles while other verbs in the frame impose different restrictions on the FE Vehicle;

- Profiling a different FE from the one profiled by the hypernym, e.g. $\{r o b: 1\}$ (Robbery) profiles the Victim, while its hypernym \{steal:1; rip off:2, rip:4\} (Theft) profiles the stolen Goods;

- Inclusion/exclusion of a causative/agentive FEs corresponding to a causative subevent in the respective pairs of frames, e.g. $\{$ break:5\} (Cause_to_fragment) and \{break:2, separate:10, fall apart:4, come apart: 1$\}$ (Breaking_apart).

Some of the types of specialisation are currently being studied as a point of departure for defining more narrow-scope frames that would allow for more precise predictions about the selectional restrictions and the syntactic realisation of FEs.

\section{Future Work}

A further goal is to enrich FN by extending its lexical coverage on the basis of the expanded mapping to synsets. Verbs which do not have correspondence among LUs (or no correspondence in a given frame) but belong to synsets that have been successfully mapped to FN frames, will be suggested as possible LUs to be included in the respective frame(s).

A venue of ongoing research is to define precise selectional restrictions on FEs and to implement them as semantic relations between a verb synset and a set of noun synonyms that satisfy these restrictions. In such a way we will enrich WN with relations between verbs and nouns corresponding to participants in their conceptual structure, particularly ones realised as arguments and adjuncts.

The developed resource may have a considerable impact on the development of methods for identification of predicate-argument structure in text, which in turn will facilitate the development of new methods for frame verification and consistency checks on FN and WN.

\section{Acknowledgments}

This study has been carried out as part of the project Towards a Semantic Network Enriched with a Variety of Semantic Relations funded by the National Science Fund of the Republic of Bulgaria under the Fundamental Scientific Research Programme (Grant Agreement 10/3 of 14.12.2016). 


\section{References}

Collin F. Baker. 2008. FrameNet, present and future. In Jonathan Webster, Nancy Ide, and Alex Chengyu Fang, editors, The First International Conference on Global Interoperability for Language Resources. City University, City University, Hong Kong.

Collin F. Baker and Christiane Fellbaum. 2009. WordNet and FrameNet as Complementary Resources for Annotation. In Proceedings of the Third Linguistic Annotation Workshop (ACL-IJCNLP '09), Association for Computational Linguistics, Stroudsburg, PA, USA. pages $125-129$.

Collin F. Baker, Charles J. Fillmore, and John B. Lowe. 1998. The Berkeley FrameNet Project. In COLINGACL '98: Proceedings of the Conference. Montreal, Canada. pages 86-90.

Collin F. Baker and Josef Ruppenhofer. 2002. FrameNet's frames vs. levin's verb classes. In J. Larson and M. Paster, editors, Proceedings of 28th Annual Meeting of the Berkeley Linguistics Society. pages 27-38.

Christiane Fellbaum. 1998. A Semantic Network of English Verbs . In Christiane Fellbaum, editor, WordNet: An Electronic Lexical Database, MIT Press, Cambridge, MA, pages 69-104.

E. Laparra and G. Rigau. 2009. Integrating WordNet and FrameNet using a knowledge-based Word Sense Disambiguation algorithm. In Proceedings of Recent Advances in Natural Language Processing (RANLP09), Borovets, Bulgaria. pages 208-213.

Egoitz Laparra and German Rigau. 2010. eXtended WordFrameNet. In Proceedings of LREC 2010. pages 1214-1219.

Svetlozara Leseva and Ivelina Stoyanova. 2019. Enhancing Conceptual Description through Resource Linking and Exploration of Semantic Relation. In Proceedings of 10th Global WordNet Conference, 23 - 27 July 2019, Wroclaw, Poland. pages 229-238.

Svetlozara Leseva, Ivelina Stoyanova, and Maria Todorova. 2018. Classifying Verbs in WordNet by Harnessing Semantic Resources. In Proceedings of CLIB 2018, Sofia, Bulgaria. pages 115-125.

George A. Miller. 1995. WordNet: A Lexical Database for English. Commun. ACM 38(11):39-41.

Martha Palmer. 2009. Semlink: Linking PropBank, VerbNet and FrameNet. In Proceedings of the Generative Lexicon Conference. 9-15.

Martha Palmer, Claire Bonial, and Diana McCarthy. 2014. SemLink+: FrameNet, VerbNet and Event Ontologies. In Proceedings of Frame Semantics in NLP: A Workshop in Honor of Chuck Fillmore (1929-2014), Baltimore, Maryland USA, June 27, 2014. Association for Computational Linguistics, pages $13-17$.
Miriam R. Petruck. 2015. The Components of FrameNet. http://

naacl.org/naacl-hlt-2015/

tutorial-framenet-data/

FNComponentsMRLP.pdf.

Miriam R. Petruck and Gerard de Melo. 2012. Precedes: A semantic relation in FrameNet. In Proceedings of the Workshop on Language Resources for Public Security Applications. pages 45-49.

Josef Ruppenhofer, Michael Ellsworth, Miriam R. Petruck, Christopher R. Johnson, C. F. Baker, and Jan Scheffczyk. 2016. FrameNet II: Extended Theory and Practice. International Computer Science Institute, Berkeley, California.

Lei Shi and Rada Mihalcea. 2005. Putting Pieces Together: Combining FrameNet, VerbNet and WordNet for Robust Semantic Parsing. In A. Gelbukh, editor, Computational Linguistics and Intelligent Text Processing. CICLing 2005. Lecture Notes in Computer Science, Springe, Berlin, Heidelbergr, volume 3406.

Sara Tonelli and Daniele Pighin. 2009. New Features for Framenet - Wordnet Mapping. In Proceedings of the Thirteenth Conference on Computational Natural Language Learning (CoNLL'09), Boulder, USA.

Robert D. Van Valin Jr. and Randy J. LaPolla. 1997. Syntax: Structure, meaning and function. Cambridge University Press. 